\title{
Tomato Plant Disease Detection using Image Processing
}

\author{
Sagar Vetal ${ }^{1}$, R.S. Khule ${ }^{2}$ \\ Department of Electronics and Telecommunication, Matoshri College of Engineering \& Research Centre, Eklahare, \\ Nashik, Savitribai Phule University of Pune, India ${ }^{1,2}$
}

\begin{abstract}
Agriculture is the major sector in India. About 58\% of the rural livelihood influenced by in agriculture. Out of which tomato is one of the common food crops in India. Due to which detection of disease on tomato plant becomes important because less susceptibility. The plants productivity gets affected if proper care is not taken. Image processing is one of upbringing technology which is helping to resolve such issues with various algorithms and techniques. Most of the diseases of tomato plant detected at initial stages as they affects leaves first. By detecting the diseases at initial stage on leaves will surely avoid impending loss. In this paper four key diseases are identified using image segmentation and Multi-class SVM algorithm. For parting of damaged area on leaves image segmentation is used and for classification of accurate disease Multi-class SVM algorithm is used. In last stage symptoms and treatment to detected diseases recommended user.
\end{abstract}

Keywords: Tomato; Plant disease; Image segmentation; Multi-class SVM.

\section{INTRODUCTION}

Tomato is one of the most caring food crops of India. This plant grown in $0.458 \mathrm{M}$ ha area with $7.277 \mathrm{M} \mathrm{mt}$ production and $15.9 \mathrm{mt} / \mathrm{ha}$ productivity. The tomato crop is cultivated in all the seasons but typically during winter and summer seasons. The crop cannot resist severe frost. It nurtures well under an average monthly temperature range of $21^{\circ}-23^{\circ} \mathrm{C}$ but commercially it may be grown at temperatures ranging from $18^{\circ} \mathrm{C}$ to $27^{\circ} \mathrm{C}$. Temperature and light intensity affect the pigmentation, fruit-sets and nutritive value of the fruits. Due to all these environments plant become very susceptible to diseases caused by fungi, bacteria, and viruses. Detection of plant disease is essential research topic. Most of the plant diseases are triggered by fungi, bacteria, and viruses. Morphological changes in leaves are the primary stage of Fungi. Bacteria are considered more embryonic than fungi in addition they generally have simpler life cycles and can be identified by morphological changes in the leaves.

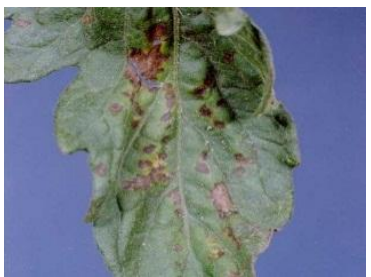

(a)

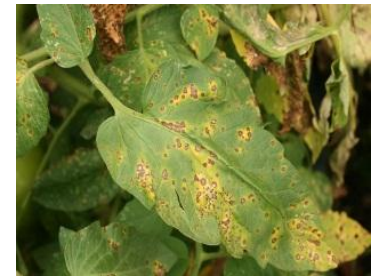

(b)

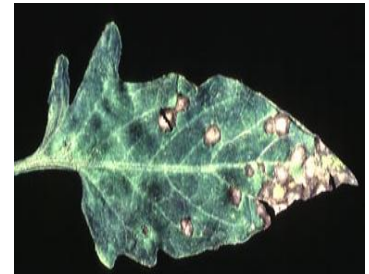

(c)

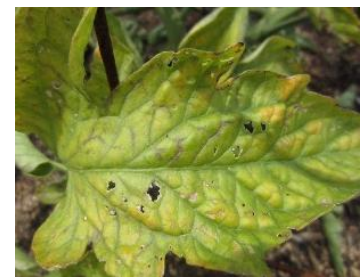

(d)

Fig. 1: Samples of tomato plant diseases; (a) Early Blight; (b) Septoria Leaf Spot; (c) Bacterial Spot (d) Iron Chlirosis

Figure 1 shows four common diseases on tomato plant. The most common method used by farmers for detection of is by naked eyes, which required experience which totally depends on knowledge of their ancestors. Other method is to refer experts which is followed by only $1 \%$ of farmers due to its higher cost. Another economical options is discussed in this paper that is using image processing.

All the diseases have common characteristics that they changes some morphological characteristics such as color or shape. These changes can be extracted through image segmentation and classification of different disease through Multiclass SVM algorithm.

\section{RELATED WORK}

There are many methods and algorithms that are being implemented on different plants for different diseases. Three steps that are followed by many of the authors. 
I. Image Preprocessing: At initial stage raw image is taken as input and smoothen through various techniques like thresholding [8], grey scale conversion [7], RGB to HIS [2], various filters like median filter[9], CIBAL color model [9] to remove noise and many more image smoothing techniques.

II. Image segmentation: the disease affected area is segmented from the leaf. For segmentation various methods are used such as k-nearest neighbor method [1], triangle threshold method and simple threshold methods [8].

III. Disease classification: For disease classification numerous algorithms are available. SVM is the most commonly used algorithm [1] [20], followed by Artificial Neural Network [5] [18], genetic programming [16] [17] [19], Histogram matching method is also used with edge detection [7].

One of the major drawback that found in SVM that it deals with binary system and can classify only 2 inputs. Histogram matching methods gives result with poor accuracy.

\section{III.PROPOSED METHODOLOGY}

Digital camera or mobiles with better resolution are used to take images of leaves of different diseases, these images are used in identifying the affected area in leafs. Various image-processing techniques are applied on them, to process raw images, to get useful image for further processing and analysis.

Algorithm written below illustrated the step by step approach for the proposed Image segmentation and disease classification processes:

1. The first step is Image acquisition, step that requires capturing an image with the help of a digital camera or mobile with better resolution.

2. Image is smoothen using Kurtosis and skewness filters.

3. Perform the image segmentation using inverse difference method to part diseases affected area from the leaf. After this stage two images are available, one with the only diseases and one with disease extracted image.

4. On this segmented image feature extraction is performed. Segmentation is the methodology in which texture and color of an image are considered, to get the unique features from the image.

Over the outdated gray-scale representation, in the visible light spectrum, an additional feature is available for image characteristic. There are three key mathematical processes in the color co-occurrence method. First is the conversion of the RGB image of a leave into HIS color space an additional feature image. After completion of this process, to generate color co-occurrence matrix, every pixel map is used, which results into three color co-occurrence matrices, one for each of H, S, I. Features called as texture features, which include Energy, Entropy, correlation and homogeneity as given in equations (1),(2),(3),(4).

Energy: Energy also means angular second moment or uniformity. The more homogeneous the image is, the larger the value. When energy levels are nearer to 1 , more the image believed to be a constant image.

$$
\text { Energy }=\sum_{\mathrm{i}=1}^{\mathrm{Ng}} \cdot \sum_{\mathrm{j}=1}^{\mathrm{Ng}} \cdot \mathrm{P}_{\mathrm{d}}(\mathrm{i}, \mathrm{j})
$$

Entropy: Entropy is a measure of randomness of intensity image.

$$
\text { Entropy }=\sum_{\mathrm{i}=1}^{\mathrm{Ng}} \cdot \sum_{\mathrm{j}=1}^{\mathrm{Ng}} \cdot \operatorname{Pd}(\mathrm{i}, \mathrm{j}) \cdot \log (\operatorname{Pd}(\mathrm{i}, \mathrm{j})) \ldots \ldots
$$

Correlation: This feature measures how correlated a pixel is to its neighborhood. It is the measure of gray tone linear dependencies in the image. Feature values range from -1 to 1 , these extremes indicating perfect negative and positive correlation respectively. $\mu \mathrm{i}$ and $\mu \mathrm{j}$ are the means oi and oj and are the standard deviations of $\operatorname{Pd}(\mathrm{i})$ and $\operatorname{Pd}(\mathrm{j})$, respectively. If the image has horizontal textures the correlation in the direction of $0^{\circ}$ degree is often larger than those in other directions. It can be calculated as

$$
\text { Correlation }=\frac{\sum_{\mathrm{i}=1}^{\mathrm{Ng}} \cdot \sum_{\mathrm{j}=1}^{\mathrm{Ng}} \cdot(1-\mu \mathrm{i})}{\text { oi. oj }}
$$

Homogeneity: Homogeneity measures the similarity of pixels. A diagonal gray level co-occurrence matrix gives homogeneity of 1 . It becomes large if local textures only have minimal changes.

$$
\text { Homogeneity }=\sum_{\mathrm{i}=1}^{\mathrm{Ng}} \cdot \sum_{\mathrm{j}=1}^{\mathrm{Ng}} \cdot \frac{\mathrm{Pd}(\mathrm{i}, \mathrm{j})}{1+|\mathrm{i}-\mathrm{j}|} \cdot \ldots
$$


TABLE 1 EXTRACTED FEATURE DATASET FOR TOMATO DISEASES USED IN DISEASE CLASSIFICATION

\begin{tabular}{|c|c|c|c|c|}
\hline Disease name & Energy & Entropy & Correlation & Homogeneity \\
\hline \multirow{4}{*}{ Early Blight } & 0.45 & 3.53 & 0.977 & 0.9746 \\
\cline { 2 - 5 } & 0.5592 & 3.0096 & 0.9225 & 0.9207 \\
\cline { 2 - 5 } & 0.3618 & 4.5526 & 0.8886 & 0.9215 \\
\cline { 2 - 5 } & 0.3938 & 4.0277 & 0.9745 & 0.9347 \\
\cline { 2 - 5 } & 0.4822 & 3.106 & 0.9019 & 0.9313 \\
\hline \multirow{3}{*}{ Septoria leaf spot } & 0.5472 & 2.6786 & 0.9017 & 0.9237 \\
\cline { 2 - 5 } & 0.6427 & 2.6788 & 0.8535 & 0.962 \\
\cline { 2 - 5 } & 0.5502 & 2.6686 & 0.9019 & 0.9257 \\
\hline \multirow{3}{*}{ Bacterial Spot } & 0.4864 & 3.4299 & 0.9824 & 0.9905 \\
\cline { 2 - 5 } & 0.7467 & 2.318 & 0.8604 & 0.9594 \\
\cline { 2 - 5 } & 0.486 & 3.43 & 0.9814 & 0.9805 \\
\hline \multirow{3}{*}{ Iron chlorosis } & 0.4884 & 3.4199 & 0.9764 & 0.9925 \\
\cline { 2 - 5 } & 0.5768 & 2.6576 & 0.8751 & 0.9639 \\
\cline { 2 - 5 } & 0.5786 & 2.6756 & 0.8571 & 0.9631 \\
\hline
\end{tabular}

5. Classification of disease: In this stage of classification, feature dataset is prepared shown in table 1. Which is an extraction of the co-occurrence features for the leaves with the analogous feature values are stored in the feature dataset. Minimum 80 sample images per disease and chosen to extract features. Variety of Extracted feature values per disease are shown in Table 1. This is the training dataset for multy-class SVM algorithm.

Then Support vector machine classifiers used for classification. Support Vector Machines only classify data into two classes. The most common technique in practice has build one-versus-rest classifiers and to select the class which classifies the test data with highest margin. Another strategy is to build a set of one-versus-one classifiers, and to choose the class that is selected by the most classifiers. As the training data set for each classifier is much reduced to binary, it encompasses building classifiers, the time for training classifiers actually decrease. However, this is unsophisticated approaches to solving multiclass problems. A better alternative to this problem is the construction of multiclass SVMs, where we build a two-class classifier over a feature vector $\Phi(\vec{x}, y)$ derived from the pair consisting of the input features and the class of the datum. At test time, the classifier chooses the class $y=\arg \cdot \max _{y^{\prime}} \vec{\omega}^{T} \Phi\left(\vec{x}, y^{\prime}\right)$. The margin during training is the gap between this value for the correct class and for the nearest other class, and so the quadratic program formulation will require this condition to be true.

$$
\forall_{\mathrm{i}} \forall_{\mathrm{y}} \neq \mathrm{y}_{\mathrm{i}} \vec{\omega}^{\mathrm{T}} \Phi\left(\overrightarrow{\mathrm{x}}_{\mathrm{i}}, \mathrm{y}_{\mathrm{i}}\right)-\vec{\omega}^{\mathrm{T}} \Phi\left(\overrightarrow{\mathrm{x}_{\mathrm{i}}}, \mathrm{y}\right) \geq 1-\xi_{\mathrm{i}}
$$

This broad method can be prolonged to give a multiclass design of various kinds of linear classifier

\section{IV.RESULT}

All the experimentation and paper related work is done in Matlab. Datasets are prepared with taking multiple images of each disease. Images are taken from different sources of camera, and at various locations near about radius of $100 \mathrm{~km}$, along with this images some standard images from different agricultural institutes are also used in preparing dataset.

The percentage accuracy is defined as the ratio of correctly recognized image samples to the total number of test image samples. The Percentage accuracy is given by Equation:

$$
\begin{aligned}
& \text { Correctly Recognized } \\
& \text { Percentage Accuracy }(\%)=\frac{\text { Image Samples }}{\text { Total Number of }} * 100
\end{aligned}
$$


Vol. 6, Issue 6, June 2017

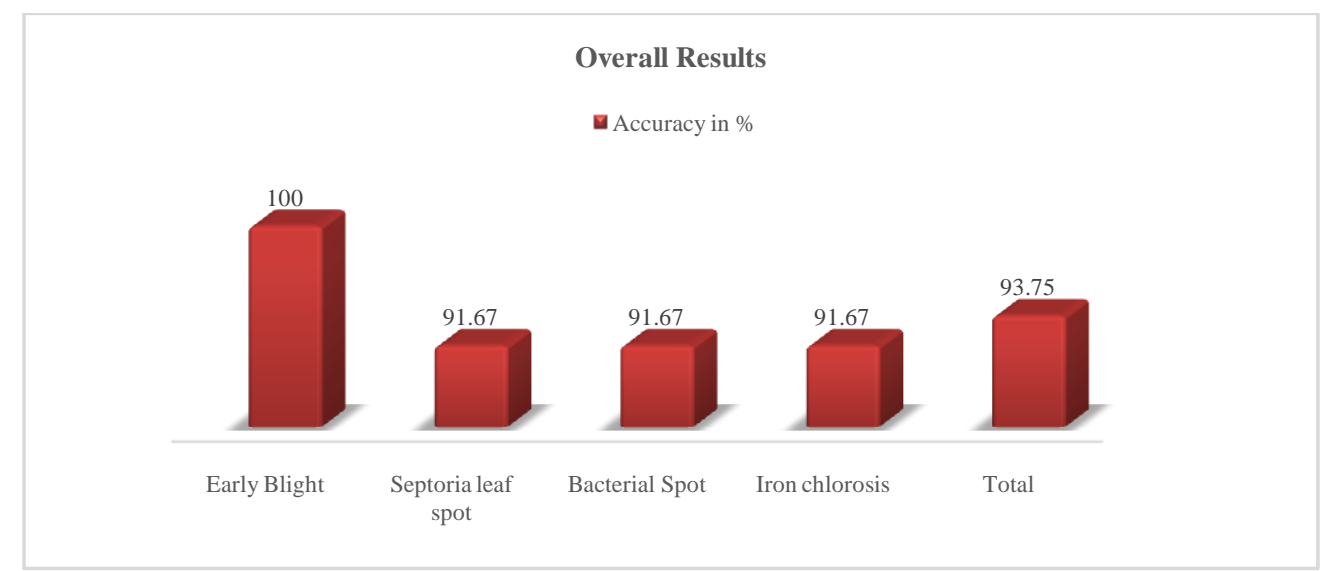

Fig. 2 Overall result

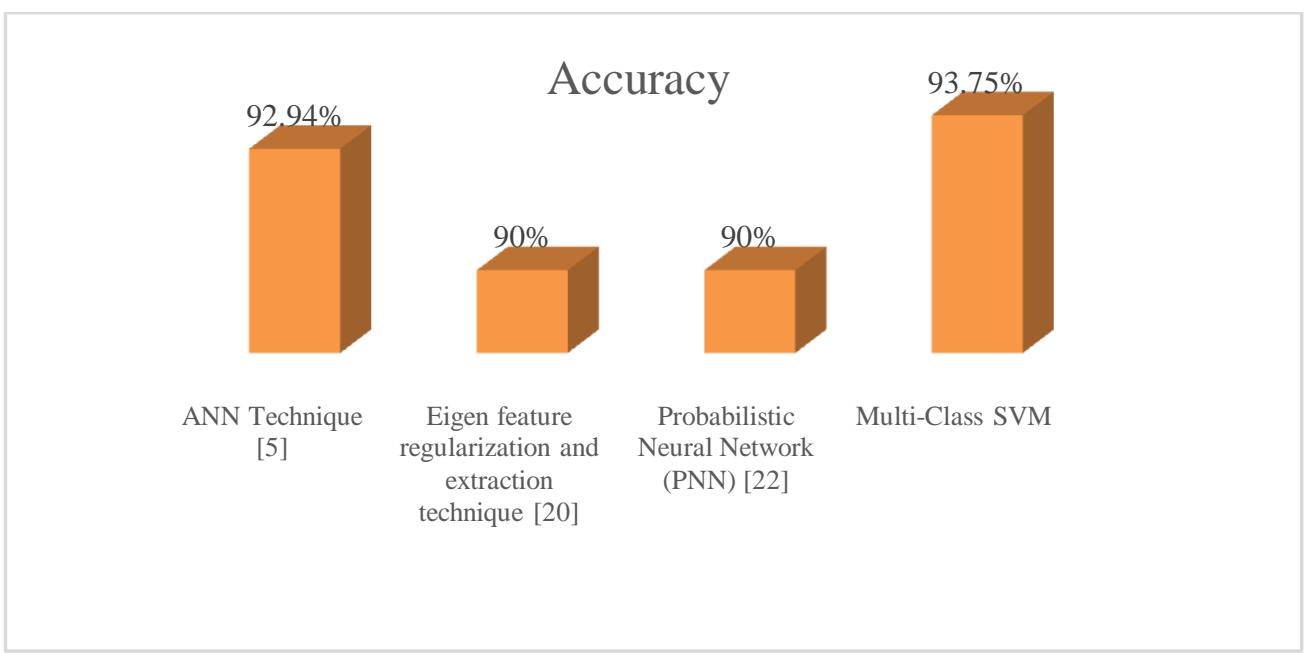

Fig.3 Comparison of Proposed technique with available techniques

\section{CONCLUSION}

For classification using Multi-class SVM model in MATLAB 15a is used. The Multi-class SVM model is trained with 80 images of one type of disease, totally algorithm is trained to detect four key diseases with 320 images. The remaining images are used for testing. Around 15\% image samples are used for validation of the designed classifier model. With different parameters, the Multi-class SVM was trained. Once the training was complete, the test data for each class of leaves was tested.

The result shown in fig. 2 obtained using Multi-class SVM classifier for 4 different diseases. The proposed system result is compared with [5][20][22] in fig.3. The result reported better classification accuracies for all the disease and percentage accuracy is $93.75 \%$.

\section{REFERENCES}

[1] Singh V, Misra A.K. Detection of plant leaf diseases using image segmentation and soft computing techniques. Info Proc-Agri (2016), http://dx.doi.org/10.1016/j.inpa.2016.10.005

[2] Dhaygude Sanjay B, Kumbhar Nitin P. Agricultural plant leaf disease detection using image processing. Int J Adv Res Electr Electron Instrum Eng 2013;2(1).

[3] Mrunalini R Badnakhe, Deshmukh Prashant R. An application of K-means clustering and artificial intelligence in pattern recognition for crop diseases. Int Conf Adv Inf Technol 2011;20. 2011 IPCSIT.

[4] Arivazhagan S, Newlin Shebiah R, Ananthi S, Vishnu Varthini S. Detection of unhealthy region of plant leaves and classification of plant leaf diseases using texture features. Agric Eng Int CIGR 2013;15(1):211-7.

[5] Kulkarni Anand H, Ashwin Patil RK. Applying image processing technique to detect plant diseases. Int J Mod Eng Res 2012; 2(5):3661-4.

[6] Bashir Sabah, Sharma Navdeep. Remote area plant disease detection using image processing. IOSR J Electron Commun Eng 2012 ; $2(6): 31-4$. ISSN: $2278-2834$.

[7] Naikwadi Smita, Amoda Niket. Advances in image processing for detection of plant diseases. Int J Appl Innov Eng Manage 2013; 2(11).

[8] Patil Sanjay B et al. Leaf disease severity measurement using image processing. Int J Eng Technol 2011;3(5):297-301.

[9] Chaudhary Piyush et al. Color transform based approach for disease spot detection on plant leaf. Int Comput Sci Telecommun 2012; 3(6). 
[10] Rathod Arti N, Tanawal Bhavesh, Shah Vatsal. Image processing techniques for detection of leaf disease. Int J Adv Res Comput Sci Softw Eng 2013; Page-3.

[11] Beucher S, Meyer F. The morphological approach to segmentation: the watershed transforms. In: Dougherty ER, editor. Mathematical morphology image processing, vol. New York: Marcel Dekker; 1993. p. 433-81.

[12] Bhanu B, Lee S, Ming J. Adaptive image segmentation using a genetic algorithm. IEEE Trans Syst Man Cybern Dec 1995; $25: 1543-67$.

[13] Bhanu B, Peng J. Adaptive integrated image segmentation and object recognition. IEEE Trans Syst Man Cybern Part C 2000; 30:427-41.

[14] Woods Keri. Genetic algorithms: colour image segmentation literature review, 2007.

[15] Vijayaraghavan Venkatesh, Garg Akhil, Wong Chee How, Tail Kang, Bhalerao Yogesh. Predicting the mechanical characteristics of hydrogen functionalized graphene sheets using artificial neural network approach. J Nanostruct Chem 2013; 3:83.

[16] Garg Akhil, Garg Ankit, Tai K. A multi-gene genetic programming model for estimating stress-dependent soil water retention curves. Comput Geosci 2014:1-12.

[17] Garg Akhil, Garg Ankit, Tai K, Sreedeep S. An integrated SRM-multi-gene genetic programming approach for prediction of factor of safety of 3-D soil nailed slopes. Eng Appl Artif Intell 2014;30:30-40.

[18] Vijayaraghavan V, Garg A, Wong CH, Tai K. Estimation of mechanical properties of nanomaterials using artificial intelligence methods. Appl Phys A 2013:1-9.

[19] Garg A, Vijayaraghavan V, Wonga CH, Tai K. Measurement of properties of graphene sheets subjected to drilling operation using computer simulation. Measurement 2014.

[20] Bernardes, Alexandre A., et al. Identification of foliar diseases in cotton crop. In: Tavares Joao Manuel RS, Renato Natal Jorge, editors. Topics in medical image processing and computational vision. Lecture Notes in Computational Vision and Biomechanics. p. 67-85.

[21] Zhen Ma, Tavares JMRS, Natal Jorge RM, A review on the current segmentation algorithms for medical images. In: 1st international conference on imaging theory and applications (IMAGAPP), Portugal; 2009. p. 135-140, ISBN: 978-989-8111-68-

[22] Panagiotis Tzionas, Stelios E. Papadakis and Dimitris Manolakis [2005] Plant leave classification based on morphological features and fuzzy surface selection technique,5thInternational Conference ON Technology and Automation ICTA'05,Thessaloniki, Greece,pp.365-370,15-16.

[23] Tavares JMRS, Image processing and analysis: applications and trends, AES-ATEMA'2010, Fifth international conference, Canada, June 27July 03; 2010. pp. 27-41, ISBN: 978-0-9780479-7-9.

[24] Glover Fred. Tabu search for nonlinear and parametric optimization (with links to genetic algorithms). Discrete Appl Math 1994;49:231-55.

[25] Al-Bashish D, Braik M, Bani-Ahmad S. Detection and classification of leaf diseases using Kmeans-based segmentation and neural-networksbased classification. Inform Technol J 2011; 10:

\section{BIOGRAPHY}

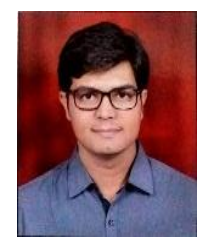

Sagar Vetal was born in Nashik, India in 1989. Received the B.E. degree in Electronics engineering from the Pune University, Pune, India, in 2011, and Perusing M.E. in VLSI and Embedded System, Electronics and Telecommunication department, Matoshri College of Engineering, Nashik, India. Currently working in the area of Plant disease detection using image processing. 